\title{
Impact of Extended Duration of Polymyxin B-Immobilized Fiber Column Direct Hemoperfusion on Hemodynamics, Vasoactive Substance Requirement, and Pulmonary Oxygenation in Patients with Sepsis: An Observational Study
}

\author{
Chieko Mitaka $^{\mathrm{a}}$ Makio Kusao $^{\mathrm{b}}$ Izumi Kawagoe ${ }^{\mathrm{a}}$ Daizoh Satoh $^{\mathrm{a}}$ Toshiaki lba $^{\mathrm{c}}$ \\ Claudio Ronco ${ }^{d}$
}

aDepartment of Anesthesiology and Pain Medicine, Juntendo University Faculty of Medicine, Tokyo, Japan;

${ }^{b}$ Department of Internal Medicine and Rheumatology, Juntendo University Faculty of Medicine, Tokyo, Japan;

'Department of Emergency and Disaster Medicine, Juntendo University Graduate School of Medicine,

Tokyo, Japan; ${ }^{d}$ Department of Nephrology Dialysis and Transplantation, International Renal Research Institute

(IRRIV), Vicenza, Italy

\section{Keywords}

Endotoxin · Sepsis · Septic shock · Polymyxin

B-immobilized fiber . Treatment duration .

Vasoactive-inotropic score

\begin{abstract}
Introduction: Polymyxin B-immobilized fiber column direct hemoperfusion (PMX-DHP) is used for patients with septic shock, and the recommended hemoperfusion period is $2 \mathrm{~h}$. However, it remains unclear whether the optimal duration is $2 \mathrm{~h}$ or longer. The purpose of this study was to compare the effects of PMX-DHP between conventional and longer duration of PMX-DHP. Methods: We retrospectively investigated 103 patients with sepsis who underwent PMX-DHP. The demographic data, routine biochemistry, microbiological data, and primary infection site were reviewed in the medical chart. The acute physiology and chronic health evaluation
\end{abstract}

karger@karger.com www.karger.com/bpu

Karger $\stackrel{\text { ' }}{5}$

BOPEN ACCESS
(C) 2021 The Author(s)

Published by S. Karger AG, Basel

This is an Open Access article licensed under the Creative Commons Attribution-NonCommercial-4.0 International License (CC BY-NC) (http://www.karger.com/Services/OpenAccessLicense), applicable to the online version of the article only. Usage and distribution for commercial purposes requires written permission.
(APACHE) II score, sequential organ failure assessment (SOFA) score, heart rate, mean arterial pressure (MAP), vasoactive-inotropic score (VIS), and $\mathrm{PaO}_{2} / \mathrm{FiO}_{2}$, at baseline and day 3, were compared between the standard group $(2 \mathrm{~h}$ of PMX-DHP) and the extended group ( $>2 \mathrm{~h}$ of PMX-DHP). $\boldsymbol{R e}$ sults: Median MAP was significantly lower and median VIS was significantly higher in the extended group at baseline $(p<0.05,0.01$, respectively) There were no significant differences in APACHE II score, SOFA score, and $\mathrm{PaO}_{2} / \mathrm{FiO}_{2}$ at baseline between the 2 groups. The increase of MAP and the decrease in VIS from baseline to day 3 were significantly greater in the extended group ( $p<0.01$, respectively). In the extended group, increase in $\mathrm{PaO}_{2} / \mathrm{FiO}_{2}$ was significantly larger in the patients who underwent $\geq 8 \mathrm{~h}$ duration than that in patients who underwent $<8 \mathrm{~h}$ duration $(p<0.01)$. The ventilator-free days, the incidence of continuous renal replacement therapy, and the 28-day mortality were not different between the groups. Discussion/Conclusions: Longer dura-
Correspondence to:

Chieko Mitaka, c-mitaka@juntendo.ac.jp 
tion of PMX-DHP was associated with the improved MAP and decreased volume of vasoactive-inotropic agents compared with the conventional duration. Eight and longer hours duration of PMX-DHP was associated with the improvement in the pulmonary oxygenation. Further studies are needed to confirm the efficacy of longer duration of PMX-DHP in patients with septic shock.

(C) 2021 The Author(s)

Published by S. Karger AG, Basel

\section{Introduction}

Sepsis is defined as the life-threatening organ dysfunction caused by a dysregulated host response to infection and septic shock is a subset in which the risk of mortality increased [1]. Despite the progress in the understanding of pathophysiology and availability of various treatments including new antibiotics, fluid therapy, and vasoactive-inotropic approaches, the patients' outcome has not been satisfactorily improved since the backgrounds of sepsis are highly heterogeneous [2]. Perhaps, a one-fits-all novel treatment for sepsis cannot be expected.

Endotoxin, an outer membrane component of Gramnegative bacteria, plays an important role in the pathogenesis of sepsis, septic shock, and sepsis-associated organ dysfunction. Endotoxin activates macrophages and other leukocytes to induce the excess cytokines production, which in turn elicits a systemic inflammatory response and leads to the endothelial dysfunction and increased vascular permeability [3]. Endotoxin adsorption by polymyxin B-immobilized fiber column (Toraymyxin ${ }^{\circledR}$ Cartridge, Toray Medical, Tokyo, Japan) direct hemoperfusion (PMX-DHP) has been used for the treatment of septic shock not only in Japan but also in Italy, Korea, Taiwan, and many other countries. Recently, the EUPHRATES trial (Evaluating the Use of Polymyxin B Hemoperfusion in a Randomized Controlled Trial of Adults Treated for Endotoxemia and Septic Shock) performed in North America has shown that PMX-DHP treatment could not reduce 28-day mortality in septic shock patients with endotoxin activity assay level $\geq 0.6$ [4]. However, a post hoc analysis has demonstrated that PMX-DHP treatment had beneficial effects on changes in mean arterial pressure (MAP), ventilator-free days to 28 days, and 28-day mortality in septic shock patients with endotoxin activity assay (EAA) level between 0.60 and 0.89 [5]. Therefore, it is hypothesized that PMXDHP treatment may have beneficial effects on the derangements of systemic circulation and ventilation in endotoxemic patients whose EAA level within the appropriate range for treatment. Consequently, a TIGRIS trial is currently underway in septic shock patients with multiple organ dysfunction score $>9$ and EAA level between 0.6 and 0.89 (ClinicalTrial.gov Identifier NCT 03901807). Another possible cause of unsatisfactory result can be the short duration of hemoperfusion. Although the instruction recommends $2 \mathrm{~h}$ of treatment [6], it remains unclear whether the optimal duration is $2 \mathrm{~h}$. Some studies performed in Japan have demonstrated that longer duration of PMX-DHP had better effects on hemodynamics and pulmonary oxygenation [7-9]. Accordingly, we planned to compare the effects of PMXDHP treatment on MAP, vasoactive-inotropic agent requirement, pulmonary oxygenation, and outcome between the conventional $(2 \mathrm{~h})$ and the longer $(>2 \mathrm{~h})$ duration of PMX-DHP.

\section{Materials and Methods}

\section{Study Design and Subjects}

We retrospectively reviewed septic patients who underwent PMX-DHP at the intensive care unit (ICU) of Juntendo University Hospital between April 2015 and March 2020. The inclusion criteria were age $\geq 18$ years, septic patients who underwent PMXDHP, and patients admitted to the ICU for at least $72 \mathrm{~h}$ of observation. Sepsis was defined as an increase in sequential organ failure assessment (SOFA) score $\geq 2$ points, and septic shock was identified by a vasopressor requirement to maintain an MAP $\geq 65 \mathrm{~mm}$ $\mathrm{Hg}$ and serum lactate level $>2 \mathrm{mmol} / \mathrm{L}$ in the absence of hypovolemia according to definitions for Sepsis-3 $[1,10]$.

The PMX-DHP treatment was performed at a blood flow rate of $40-100 \mathrm{~mL} / \mathrm{min}$. The blood flow was regulated depending on the patients' condition, and the flow was targeted $100 \mathrm{~mL} / \mathrm{min}$ as long as the circulation was stable. Nafamostat mesylate (AY Pharmaceuticals Co., Ltd, Tokyo, Japan), a protease inhibitor, was administered at $30-40 \mathrm{mg} / \mathrm{h}$ as an anticoagulant, and this procedure is popular in Japan. Basically, the duration of PMX-DHP treatment was determined by consensus among the attending physicians, intensivists, and apheresis unit doctors. The duration was set for $2 \mathrm{~h}$ in the early phase of the study. However, since we learned that endotoxin removal increases along with the hemoperfusion period theoretically and experienced that some cases responded to the longer period of perfusion even the circulatory disturbance was severer, we expanded the duration in the later phase of the study.

The study protocol was reviewed and approved by the Ethics Committee of Juntendo University Hospital (approval number 19291). This research complied with guidelines for human studies and was conducted ethically in accordance with the World Medical Association Declaration of Helsinki. The need for patient approval and informed consent was waived due to the retrospective nature of the study. Instead, the protocol summary was publicized on the university website clearly informing of the patients' right to refuse participation. 
Table 1. Patients' characteristics at baseline

\begin{tabular}{lccc}
\hline Parameter & $\begin{array}{l}\text { All patients } \\
(n=103)\end{array}$ & $\begin{array}{l}\text { Standard group } \\
(n=58)\end{array}$ & $\begin{array}{l}\text { Extended group } \\
(n=45)\end{array}$ \\
\hline Age, years old & $71(62,77)$ & $71(64,78)$ & $70(61,76)$ \\
Gender, male $(\%)$ & $59(57)$ & $35(60)$ & $24(53)$ \\
Heart rate, bpm & $104(91,121)$ & $105(92,118)$ & $104(90,130)$ \\
$\mathrm{MAP}, \mathrm{mm} \mathrm{Hg}$ & $61(53,73)$ & $66(58,76)$ & $58(50,79)$ \\
VIS & $10(2.2,22.1)$ & $6.8(0,17.3)$ & $14(7,30)$ \\
Incidence of VIS $\geq 20$ & $32(31)$ & $13(22)$ & $19(42)$ \\
Respiratory rate, /min & $26(21,33)$ & $26(23,33)$ & $25(20,34)$ \\
Lactate, $\mathrm{mmol} / \mathrm{L}$ & $2.85(1.80,5.43)$ & $2.40(1.90,5.59)$ & $3.46(1.8,5.43)$ \\
PaO $/$ FiO, $\mathrm{mm} \mathrm{Hg}$ & $235(161,310)$ & $263(164,336)$ & $214(133,245)$ \\
APACHE $\mathrm{II} \mathrm{score}$ & $18(14,23)$ & $18(12,22)$ & $18(16,24)$ \\
SOFA score & $8(6,11)$ & $8(6,11)$ & $8(7,11)$ \\
WBC count, $\times 10^{3} / \mu \mathrm{L}$ & $10.3(5.55,19.65)$ & $10.3(4.5,17.6)$ & $11.4(5.9,21.5)$ \\
Hematocrit, $\%$ & $29.8(25.7,34.3)$ & $29.7(26.0,36.1)$ & $29.9(25.0,32.9)$ \\
Platelet, $\times 10^{3} / \mu \mathrm{L}$ & $101(61,159)$ & $94(56,156)$ & $122(66,162)$ \\
Bilirubin, $\mathrm{mg} / \mathrm{dL}$ & $1.06(0.59,1.84)$ & $1.27(0.60,1.92)$ & $1.02(0.57,1.64)$ \\
Creatinine, $\mathrm{mg} / \mathrm{dL}$ & $1.66(1.17,3.09)$ & $2.07(1.17,3.53)$ & $1.58(1.17,2.41)$ \\
CRP, mg/dL & $14.8(6.9,20.7)$ & $14.5(6.8,21.2)$ & $15.4(8.7,20.1)$ \\
Procalcitonin, $\mathrm{ng} / \mathrm{mL}$ & $32.4(7.8,82.6)$ & $24.4(6.6,50.4)$ & $38.7(18.6,109.8)$ \\
\hline
\end{tabular}

The qualitative data are shown as number (percentage) and the quantitative data are shown as medians (interquartile ranges). PMXDHP, polymyxin B-immobilized fiber column direct hemoperfusion; MAP, mean arterial pressure; VIS, vasoactive-inotropic score; APACHE II, acute physiology and chronic health evaluation II; SOFA, sequential organ failure assessment; WBC, white blood cell; CRP, C-reactive protein. $p$ value, Standard group versus Extended group.

\section{Data Collection}

The following data of all patients were obtained from medical records: demographic information including age, sex, laboratory data including white blood cell count, hematocrit, platelet count, levels of total bilirubin, creatinine, C-reactive protein, procalcitonin, and lactate, primary infection site, bacterial culture results, heart rate, MAP, dose of vasoactive-inotropic agent, respiratory rate, $\mathrm{PaO}_{2} / \mathrm{FiO}_{2}$ ratio, duration and frequency of PMX-DHP, and concomitant use of continuous renal replacement therapy (CRRT) and mechanical ventilation. In the present study, 12 patients received vasopressin in addition to norepinephrine. Therefore, the dose of vasoactive-inotropic agent was expressed as vasoactiveinotropic score (VIS) [11] and calculated as follows:

VIS = Dopamine dose $(\mu \mathrm{g} / \mathrm{kg} / \mathrm{min})+$ Dobutamine dose $(\mu \mathrm{g} / \mathrm{kg} /$ $\min )+100 \times$ Epinephrine dose $(\mu \mathrm{g} / \mathrm{kg} / \mathrm{min})+10 \times$ Milrinone dose $(\mu \mathrm{g} / \mathrm{kg} / \mathrm{min})+10,000 \times$ Vasopressin dose (units $/ \mathrm{kg} / \mathrm{min})+100 \times$ Norepinephrine dose $(\mu \mathrm{g} / \mathrm{kg} / \mathrm{min})$.

The severity of illness was assessed using acute physiology and chronic health evaluation II score [12], and the severity of organ dysfunction was assessed by SOFA score [10]. To assess the impact on the efficacy of longer duration of PMX-DHP treatment, we divided the patients into 2 groups: the standard $(2 \mathrm{~h})$ group and the extended $(>2 \mathrm{~h})$ group and compared the abovementioned parameters between the 2 groups. Changes in MAP, VIS, $\mathrm{PaO}_{2} / \mathrm{FiO}_{2}$, SOFA score, lactate level, and platelet count from baseline to day 3 were also compared between the 2 groups. In addition, ventilator-free days to 28 days, rate of CRRT, and the 28-day mortality were compared between the 2 groups.

\section{Statistical Analysis}

Quantitative data are expressed as the median and interquartile range (IQR). The intergroup differences were compared using the Mann-Whitney $U$ test. Categorical data are expressed as absolute values and percentages, and they were analyzed using the $\chi^{2}$ test. A $p$ value of $<0.05$ was considered statistically significant for all comparisons.

\section{Results}

There were 103 septic patients who underwent PMXDHP: the standard group $(n=58)$ and the extended group $(n=45)$. The patients' characteristics at baseline are shown in Table 1. The number of septic shock patients in the standard group and in the extended group was 41 (71\%) and 45 (100\%), respectively. At baseline, the median MAP in the extended group was significantly lower $(p<0.05)$, and the median VIS in the extended group was significantly higher than the standard group $(p<0.01)$. The number of patients with VIS $\geq 20$ in the standard group and in the extended group was 13 (22\%) and 19 (42\%), respectively. Thus, the number of patients with VIS $\geq 20$ in the extended group was significantly larger 
Table 2. Site of infection, type of microorganisms, and rate of bacteremia

\begin{tabular}{lccc}
\hline Parameter & $\begin{array}{l}\text { All patients } \\
(n=103)\end{array}$ & $\begin{array}{l}\text { Standard group } \\
(n=58)\end{array}$ & $\begin{array}{l}\text { Extended group } \\
(n=45)\end{array}$ \\
\hline Site of infection, case (\%) & & & \\
$\quad$ Abdomen & $42(41)$ & $24(42)$ & $18(40)$ \\
$\quad$ Urinary tract & $17(16)$ & $6(10)$ & $11(25)$ \\
$\quad$ Blood & $16(15)$ & $11(19)$ & $5(11)$ \\
Lung & $9(9)$ & $7(12)$ & $2(4)$ \\
Skin and soft tissue & $7(7)$ & $3(5)$ & $4(9)$ \\
$\quad$ Unknown & $12(12)$ & $7(12)$ & $5(11)$ \\
Microorganisms, case (\%) & & & \\
$\quad$ Gram-negative & $61(59)$ & $33(57)$ & $7(62)$ \\
$\quad$ Gram-positive & $13(13)$ & $6(10)$ & $4(9)$ \\
Mixed & $11(11)$ & $7(12)$ & $6(13)$ \\
No growth & $18(17)$ & $12(21)$ & $25((56)$ \\
Bacteremia, case (\%) & & & $20(44)$ \\
Yes & $58(56)$ & $33(57)$ & \\
No & $45(44)$ & $25(43)$ & \\
\hline
\end{tabular}

Data are shown as number (percentage).

Table 3. Changes in various parameters from baseline to day 3

\begin{tabular}{|c|c|c|c|c|}
\hline Parameter & $\begin{array}{l}\text { Standard group } \\
(n=58)\end{array}$ & $\begin{array}{l}\text { Extended group } \\
(n=45)\end{array}$ & & $p$ value \\
\hline$\triangle \mathrm{MAP}, \mathrm{mm} \mathrm{Hg}$ & $20(8,31)$ & $26(16,38)$ & & 0.03 \\
\hline$\Delta \mathrm{VIS}$ & $-1(-10,0)$ & $-10(-21,-3.9)$ & & $<0.01$ \\
\hline \multirow{2}{*}{$\Delta \mathrm{PaO}_{2} / \mathrm{FiO}_{2}, \mathrm{~mm} \mathrm{Hg}$} & \multirow{2}{*}{$60(1,102)$} & \multicolumn{2}{|c|}{$71(22,122)$} & 0.48 \\
\hline & & $\begin{array}{r}\text { duration }<8 \mathrm{~h} \\
61(10,82)\end{array}$ & $\begin{array}{l}\geq 8 \mathrm{~h} \\
100(74,168)\end{array}$ & $<0.01$ \\
\hline$\triangle$ SOFA score & $-2(-4,-1)$ & $-3(-5,-1)$ & & 0.18 \\
\hline$\Delta$ Lactate, $\mathrm{mmol} / \mathrm{L}$ & $-1.57(-3.45 .-0.39)$ & $-1.74(-3.51,-0.76)$ & & 0.60 \\
\hline$\Delta$ Platelet, $\times 10^{3} / \mu \mathrm{L}$ & $-25.5(-47.3,-1.8)$ & $-34.0(-62.0,-15.0)$ & & 0.15 \\
\hline
\end{tabular}

Data are shown as median (interquartile range). MAP, mean arterial pressure; VIS, vasoactive-inotropic score; SOFA, sequential organ failure assessment; $\triangle \mathrm{MAP}, \mathrm{MAP}$ on day3 - MAP at baseline; $\triangle$ VIS, VIS on day 3 - VIS at baseline; $\Delta \mathrm{PaO}_{2} / \mathrm{FiO}_{2}, \mathrm{PaO}_{2} / \mathrm{FiO}_{2}$ on day $3-\mathrm{PaO}_{2} / \mathrm{FiO}_{2}$ at baseline; $\Delta$ SOFA score, SOFA score at baseline SOFA score on day3; $\Delta$ Lactate, lactate level at baseline - lactate level on day $3 ; \Delta$ Platelet, platelet count on day 3 - platelet count at baseline.

compared with the standard group $(p<0.05)$. At baseline, there were no significant differences in respiratory rate, lactate level, $\mathrm{PaO}_{2} / \mathrm{FiO}_{2}$, acute physiology and chronic health evaluation II score, SOFA score, white blood cell count, hematocrit, platelet count, and levels of total bilirubin, creatinine, C-reactive protein, and procalcitonin between the 2 groups.

The site of infection, type of microorganisms, and rate of bacteremia are shown in Table 2 . The most common site of infection in all patients was abdomen (41\%), followed by urinary tract (16\%) and blood (15\%). Of those who had positive cultures, Gram-negative organisms ac- counted for 59\% and Gram-positive organisms accounted for $13 \%$. The proportion of patients where all obtained cultures showed no growth of bacteria was $17 \%$. Blood cultures were positive in $56 \%$ of the patients.

Changes in MAP, VIS, $\mathrm{PaO}_{2} / \mathrm{FiO}_{2}$, SOFA score, lactate level, and platelet count from baseline to day 3 are shown in Table 3. Changes in MAP and VIS in the extended group were significantly larger compared with the standard group ( $p<0.05,0.01$, respectively). Changes in $\mathrm{PaO}_{2} / \mathrm{FiO}_{2}$, SOFA score, lactate level, and platelet count from baseline to day 3 were not statistically different between the 2 groups. However, among the extended group, 
Table 4. Implementation of PMX-DHP, CRRT, and mechanical ventilation and 28-day mortality

\begin{tabular}{|c|c|c|c|c|}
\hline Parameter & $\begin{array}{l}\text { All patients } \\
(n=103)\end{array}$ & $\begin{array}{l}\text { Standard group } \\
(n=58)\end{array}$ & $\begin{array}{l}\text { Extended group } \\
(n=45)\end{array}$ & $p$ value \\
\hline \multicolumn{5}{|l|}{ PMX-DHP } \\
\hline Two sessions, $n(\%)$ & $90(87)$ & $47(81)$ & $43(96)$ & 0.02 \\
\hline Total duration, $\mathrm{h}$ & $4(4,6)$ & $4(4,4)$ & $6(5,10)$ & $<0.001$ \\
\hline Total amount of blood treated, $\mathrm{mL}$ & $19,299(18,030,23,400)$ & $19,200(16,800,19,200)$ & $26,400(21,600,42,450)$ & $<0.001$ \\
\hline CRRT, case $n(\%)$ & $51(50)$ & $31(53)$ & $20(44)$ & 0.36 \\
\hline Mechanical ventilation, case $n(\%)$ & $42(41)$ & $19(33)$ & $23(51)$ & 0.06 \\
\hline Ventilator-free days to 28 days & $20(15,23)$ & $20(14,23)$ & $20(17,22)$ & 0.96 \\
\hline 28-day mortality, $n(\%)$ & $20(19)$ & $9(16)$ & $11(24)$ & 0.25 \\
\hline
\end{tabular}

The qualitative data are shown as number (percentage), and the quantitative data are shown as medians (interquartile range). PMXDHP, polymyxin B-immobilized fiber column direct hemoperfusion; CRRT, continuous renal replacement therapy; ICU, intensive care unit.

the patients who underwent $\geq 8 \mathrm{~h}$ of duration $(n=20)$ revealed median (IQR) change in $\mathrm{PaO}_{2} / \mathrm{FiO}_{2}$ was $100 \mathrm{~mm}$ $\mathrm{Hg}(74 \mathrm{~mm} \mathrm{Hg}, 168 \mathrm{~mm} \mathrm{Hg}$ ) and the rise was significantly larger than that in the patients who underwent $<8 \mathrm{~h}$ duration $(n=25)(61 \mathrm{~mm} \mathrm{Hg}[10 \mathrm{~mm} \mathrm{Hg}, 82 \mathrm{~mm} \mathrm{Hg}]$, $p<0.01$ ). In the extended group, 11 patients with $\mathrm{PaO}_{2} /$ $\mathrm{FiO}_{2} \leq 200 \mathrm{~mm} \mathrm{Hg}$ at baseline had concomitant moderate or severe acute respiratory distress syndrome [13]. The median (IQR) $\mathrm{PaO}_{2} / \mathrm{FiO}_{2}$ at baseline in the patients who underwent $\geq 8 \mathrm{~h}$ duration was $133 \mathrm{~mm} \mathrm{Hg}$ (78 $\mathrm{mm} \mathrm{Hg}$, $215 \mathrm{~mm} \mathrm{Hg}$ ), and this was significantly lower than that in the patients who underwent $<8 \mathrm{~h}$ duration $(230 \mathrm{~mm} \mathrm{Hg}$ [211 mm Hg, $347 \mathrm{~mm} \mathrm{Hg}$, $p<0.001$ ).

The implementation of PMX-DHP, CRRT, and mechanical ventilation and 28-day mortality are shown in Table 4. A second session was not carried out when the patient's hemodynamics improved or deceased after the first session. As a result, the incidence of 1 session was significantly lower and that the 2 sessions were significantly higher in the extended group $(p<0.05)$. The median total duration of PMX-DHP was significantly $(p<$ 0.01 ) longer in the extended group than in the standard group. The median total amount of blood treated was significantly $(p<0.001)$ larger in the extended group than in the standard group. The treatment duration greater than $2 \mathrm{~h}$ was applied to both hemoperfusions in 23 patients. While 10 patients received extended hemoperfusion for the first session only, and 12 patients received for the second session only. There were no significant differences in the interval between ICU admission and PMX-DHP initiation, rate of CRRT and mechanical ventilation, venti- lator-free days to 28 days, and the 28-day mortality between the 2 groups. Mortality at 28 days based on site of infection was abdomen (standard group 3/9 [33\%] vs. extended group 4/11 [36\%]), lung (standard group 2/9 [22\%] vs. extended group 1/11 [9\%]), urinary tract (standard group 0/9 [0\%] vs. extended group 3/11 [27\%]), blood (standard group 1/9 [11\%] vs. extended group 1/11 [9\%]), skin and soft tissue (standard group 2/9 [22\%] vs. extended group $0 / 11[0 \%]$ ), and unknown (standard group 1/9 [11\%] vs. extended group 2/11 [18\%]).

\section{Discussion/Conclusion}

The major finding in the present study was that the longer duration of PMX-DHP treatment was associated with significant hemodynamic improvement and reduction of vasoactive-inotropic agent requirement. At baseline, MAP was significantly lower and VIS was significantly higher in the extended group than the standard group. High VIS was empirically defined as $\geq 20$ and maximum VIS $\geq 20$ has been shown to predict poor clinical outcomes [11], and the patients with high VIS were $22 \%$ in the standard group and $42 \%$ in the extended group in the present study. Thus, it was estimated that a longer duration of PMX-DHP tended to be applied in patients who required more vasoactive-inotropic agents to maintain hemodynamics. Some previous studies performed in Japan have shown that longer duration of PMX-DHP had better performance on hemodynamics [7-9]. The present study confirmed the above results, and the effects on hemody- 
namics were better in the extended group. These findings suggest that a longer duration of $>2 \mathrm{~h}$ of PMX-DHP may help the recovery of the systemic circulation and contribute to decreasing the vasoactive-inotropic agent use, which may lead to the prevention of life-threatening organ failure. Total amount of blood treatment was significantly larger in the extended group than in the standard group. Therefore, longer duration as well as the larger amount of hemoperfusion might relate to the effects. With respect to the hemoperfusion period, the previous in vitro study using calf serum has demonstrated that the PMX-DHP could reduce the endotoxin level considerably in $2 \mathrm{~h}$ of circulation [6], and this is the rationale for the $2 \mathrm{~h}$ standard duration of the PMX-DHP treatment. However, we have previously reported that a longer duration of PMX-DHP decreased norepinephrine dose and decreased circulating adhesion molecules, which resulted in the improvement of hemodynamics and pulmonary oxygenation [7].

In the present study, the subanalysis of the extended group clarified that an increase in $\mathrm{PaO}_{2} / \mathrm{FiO}_{2}$ from baseline to day 3 was significantly larger in patients who underwent $\geq 8$ h duration than that in the patients who underwent $<8$ $\mathrm{h}$ duration. This finding suggests that a longer duration of PMX-DHP is necessary to improve pulmonary oxygenation than to improve hemodynamic decompensation. The reason why there was no difference in the pulmonary oxygenation between the standard group and the total extended group is probably that more than half of patients in the extended group were undergone $<8 \mathrm{~h}$ duration.

Yamashita et al. [8] have also shown that longer duration of PMH-DHP improved hemodynamics and pulmonary oxygenation in 37 patients with septic shock. Miyamoto et al. [9] have reported in their retrospective study that $12 \mathrm{~h}$ duration of PMX-DHP $(n=18)$ significantly increased MAP and decreased the vasopressor dependency index compared with $2 \mathrm{~h}$ duration of PMX-DHP ( $n=$ 18). Furthermore, Kawazoe et al. [14] have demonstrated in a subanalysis of the DESIRE trial, a Dexmedetomidine for Sepsis in Intensive Care Unit Randomized Evaluation study, that the 28 -day mortality rate was $7 / 22(31.8 \%)$ in the standard group and $0 / 14(0 \%)$ in the extended group. Although all aforementioned studies including ours indicated the same trends, they are all observational studies and a prospective controlled study is warranted.

With respect to the further extension of the duration, we have demonstrated that the median endotoxin removal rate was $74.4 \%$ after $24 \mathrm{~h}$ PMX-DHP in 19 septic shock patients in other case series study, suggesting that $24 \mathrm{~h}$ treatment was effective in removing endotoxin [15]. Therefore, the duration may be able to be extended to as long as $24 \mathrm{~h}$.
Romaschin et al. [16] performed an in vitro experiment to examine the endotoxin adsorption capacity of PMXDHP column (PMX-20R) using a closed-circuit system. A single introduction of endotoxin-activated bovine serum or plasma was recirculated through PMX-20R using a roller pump at $100 \mathrm{~mL} / \mathrm{min}$ for $4 \mathrm{~h}$. The total endotoxin amount given to the circuit was $15 \mu \mathrm{g}$, and the initial concentration in the perfusate was $10 \mathrm{ng} / \mathrm{mL}$. After $4 \mathrm{~h}$ of perfusion, the endotoxin concentration decreased to 2-3 ng/ $\mathrm{mL}$, and the residual endotoxin in the perfusate was approximately $3 \mu \mathrm{g}$. Therefore, the amount of endotoxin removal was $80 \%$ of the administered volume and $12 \mu \mathrm{g}$. Eventually, endotoxin adsorption ratios were $60-80 \%$ for first 2 -h hemoperfusions and reducing to $40 \%$ thereafter. These findings indicated the possibility of continuous endotoxin removal by 24-h PMX-DHP in the future. Perhaps, continuous removal is important because endotoxin is suspected to be continuously derived from the lysed bacteria. Antibiotics induce bacteria to cell lysis and stimulate endotoxin release [17], and antibiotic-induced endotoxin release varies depending on the antibiotic class, the type of bacteria, and the site of antibiotic action [18, 19]. Moreover, endotoxin can be released into the bloodstream by translocation because of the gut barrier disruption in critically ill patients [20]. In such cases, a longer duration of PMX-DHP treatment may particularly be useful.

Early recovery from circulatory shock is vital in sepsis management and De Rosa et al. [21] emphasized the importance of early initiation of PMX-DHP. In the present study, the median interval from ICU admission to PMXDHP initiation was $2.5 \mathrm{~h}$ in both groups. The most commonly observed adverse events in PMX-DHP are thrombocytopenia, transient hypotension, and allergic reactions [22]. In the present study, transient hypotension or allergic reactions was not recognized in both groups. Although platelet count tended to decrease after PMX-DHP treatment in both groups, change in platelet count from baseline to day 3 was not significantly different between the 2 groups.

The strengths of the present study are as follows. First, the number of patients is larger than the previous studies that examined the effects of extended PMX-DHP. Second, we compared the changes in hemodynamics, vasoactive substance requirement, and pulmonary oxygenation from baseline to 3 days after the treatment. This method allowed us to compare sustained effect of PMX-DHP on various clinically important parameters between the 2 groups. Third, although both the improvements in hemodynamics and oxygenation have been reported previously, our study first reported the latter effect was recognized 
after $8 \mathrm{~h}$ of duration. On the other hand, there are several limitations to this study. First, the present study is retrospective and single-center study, and the duration of PMX-DHP was not prefixed meaning that we could not waived the possibility that PMX-DHP was discontinued because the effect was not recognized. However, it was appreciated that the current study demonstrated similar results with the previous reports. Second, the level of endotoxin was not assessed beforehand. Third, $57 \%$ of patients in the standard group and $62 \%$ of patients in the extended group were the Gram-negative infection. Although the incidence was higher in the extended group, there was no significant difference in the rate of Gram-negative infection. Finally, there was no PMX-DHP control group.

In conclusion, longer duration of PMX-DHP was significantly associated with the improved MAP and decreased dose of vasoactive-inotropic agents compared with the conventional duration of PMX-DHP. Eight or more hours of duration of PMX-DHP was associated with the increased pulmonary oxygenation. There were no significant differences in the 28-day mortality between the 2 groups. Further prospective studies are warranted to confirm the efficacy of longer duration of PMX-DHP in patients with sepsis.

\section{Acknowledgements}

The authors would like to thank Go Murayama and Takuya Nemoto for technical assistance as staff members of the apheresis unit of Juntendo University Hospital.

\section{Statement of Ethics}

The study protocol was reviewed and approved by the Ethics Committee of Juntendo University Hospital (approval number 19291). This research complied with guidelines for human studies and was conducted ethically in accordance with the World Medical Association Declaration of Helsinki. The need for patient approval and informed consent was waived due to the retrospective nature of the study. Instead, protocol summary was publicized on the university Web site clearly informing of the patients' right to refuse participation.

\section{Conflict of Interest Statement}

In the last 3 years, Claudio Ronco has been consulting or part of advisory boards for ASAHI, Astute, Baxter, Biomerieux, B. Braun, Cytosorbents, ESTOR, FMC, GE, Jafron, Medtronic, and Toray. The other authors have no conflicts of interest to declare.

\section{Funding Sources}

There was no funding for this study.

\section{Author Contributions}

All authors contributed to the conception and design of the study. C.M., M.K., and I.K. were involved in data collection. C.M. and D.S. analyzed and interpreted the data and performed the statistical analysis. C.M. drafted the manuscript. T.I. and C.R. were involved in the critical revision of the manuscript. All authors read and approved the final manuscript.

\section{References}

1 Singer MS, Deutschman CS, Seymour CW, Shankar-Hari M, Annane D, Bauer M, et al. The third international consensus definition for sepsis and septic shock (Sepsis-3). JAMA. 2016 Feb;315(8):801-10.

2 Hotchkiss RS, Moldawer LL, Opal SM, Reinhart K, Turnbull IR, Vincent JL. Sepsis and septic shock. Nat Rev Dis Primers. 2016 Jun; 2:16045.

3 Peters K, Unger RE, Brunner J, Kirkpatrick CJ. Molecular basis of endothelial dysfunction in sepsis. Cardiovasc Res. 2003 Oct;60(1): 49-57.

4 Dellinger RP, Bagshaw SM, Antonelli M, Foster DM, Klein DJ, Marshall JC, et al. Effect of targeted polymyxin B hemoperfusion on 28day mortality in patients with septic shock and elevated endotoxin level. The EUPHRATES randomized clinical trial. JAMA. 2018 Oct;320(14):1455-63.
5 Klein DJ, Foster D, Walker PM, Bagshaw SM, Mekonnen H, Antonelli M. Polymyxin B hemoperfusion in endotoxemic septic shock patients without extreme endotoxemia: a post hoc analysis of the EUPHRATES trial. Intensive Care Med. 2018 Dec;44(12):2205-12.

6 Shoji H. Extracorporeal endotoxin removal for the treatment of sepsis: endotoxin adsorption cartridge (Toraymyxin). Ther Apher Dial. 2003 Feb;7(1):108-14.

7 Mitaka C, Tsuchida N, Kawada K, Nakajima Y, Imai T, Sasaki S. A longer duration of polymyxin B-immobilized fiber column hemoperfusion improves pulmonary oxygenation in patients with septic shock. Shock. 2009 Nov;32(5):478-83.

8 Yamashita C, Hara Y, Kuriyama N, Nakamura T, Nishida O. Clinical effects of a longer duration of polymyxin B-immobilized fiber column direct hemoperfusion therapy for severe sepsis and septic shock. Ther Apher Dial. 2015 Aug;19(4):316-23.
9 Miyamoto K, Kawazoe Y, Kato S. Prolonged direct hemoperfusion using a polymyxin $\mathrm{B}$ immobilized fiber cartridge provides sustained circulatory stabilization in patients with septic shock: a retrospective observational before-after study. J Intensive Care. 2017Feb 20;5:19.

10 Vincent JL, de Mendonça A, Cantraine F, Moreno R, Takala J, Suter PM, et al. Use of the SOFA score to assess the incidence of organ dysfunction/failure in intensive care units: results of a multicenter, prospective study. Working group on "sepsis-related problems" of the European Society of Intensive Care Medicine. Crit Care Med. 1998 Nov;26(11):1793-800.

11 Gaies MG, Jeffries HE, Niebler RA, Pasquali SK, Donohue JE, Yu S, et al. Vasoactive-inotropic score (VIS) is associated with outcome after infant cardiac surgery: an analysis from the pediatric cardiac critical care consortium and virtual PICU system registries. Pediatr Crit Care Med. 2014 Jul;15(16):529-37. 
12 Knaus WA, Draper EA, Wagner DP, Zimmerman JE. APACHE II: a severity of disease classification system. Crit Care Med. 1985 Oct;13(10):818-29.

13 ARDS Definition Task Force; Ranieri VM, Ranieri VM, Rubenfeld GD, Thompson BT, Ferguson ND, et al. Acute respiratory distress syndrome: the Berlin definition. JAMA. 2012 Jun;307(23):2526-33.

14 Kawazoe Y, Sato T, Miyagawa N, Yokokawa Y, Kushimoto S, Miyamoto K, et al. Mortality effects of prolonged hemoperfusion therapy using a polymyxin B-immobilized fiber column for patients with septic shock: a subanalysis of the DESIRE trial. Blood Purif. 2018 Aug;46(4):309-14.
15 Mitaka C, Fujiwara N, Yamamoto M, Toyofuku T, Haraguchi G, Tomita M. Polymyxin B-immobilized fiber column hemoperfusion removes endotoxin throughout a 24 -h treatment period. J Crit Care. 2014 Oct;29(5):72832.

16 Romaschin AD, Obiezu-Forster CV, Shoji H, Klein DJ. Novel insights into the direct removal of endotoxin by polymyxin B hemoperfusion. Blood Purif. 2017 Jun;44(3):193-7.

17 Shenep JL, Flynn PM, Barrett FF, Stidham GL, Westenkirchner DF. Serial quantitation of endotoxemia and bacteremia during therapy for gram-negative bacterial sepsis. J Infect Dis. 1988 Mar;157(3):565-8.
18 Eng RH, Smith SM, Fan-Havard P, Ogbara T. Effect of antibiotics on endotoxin release from gram-negative bacteria. Diagn Microbiol Infect Dis. 1993 Mar-Apr;16(3):185-9.

19 Holzheimer RG. Antibiotic induced endotoxin release and clinical sepsis: a review. J Chemother. 2001 Nov;13(1):159-72.

20 Grunfeld C, Feingold KR. Endotoxin in the gut and chylomicrons: translocation or transportation? J Lipid Res. 2009 Jan;50(1):1-2.

21 De Rosa S, Villa G, Ronco C. The golden hour of polymyxin $\mathrm{B}$ hemoperfusion in endotoxic shock: the basis for sequential extracorporeal therapy in sepsis. Artif Organs. 2020 Feb; 44(2):184-6.

22 Ronco C, Klein DJ. Polymyxin B hemoperfusion: a mechanistic perspective. Crit Care. 2014 Jun;18(3):309. 